\title{
Brief Analysis on the Developmental and Creative Approach of Large-Scale Lacquerware Crafts with Ethnic Characteristics
}

\author{
Chaoyi You, Yu Han*, Qianying Wei \\ School of Urban Planning and Architecture, Southwest Minzu University, Chengdu, China \\ Email: *651645399@qq.com
}

How to cite this paper: You, C. Y., Han, Y., \& Wei, Q. Y. (2019). Brief Analysis on the Developmental and Creative Approach of Large-Scale Lacquerware Crafts with Ethnic Characteristics. Art and Design Review, 7, 115-123.

https://doi.org/10.4236/adr.2019.72010

Received: May 5, 2019

Accepted: May 20, 2019

Published: May 23, 2019

Copyright ( 2019 by author(s) and Scientific Research Publishing Inc. This work is licensed under the Creative Commons Attribution International License (CC BY 4.0).

http://creativecommons.org/licenses/by/4.0/

\begin{abstract}
Lacquer wares result with the ethnic characteristics. From the emergence in the Neolithic Age to the common utilization in Qin and Han Dynasties, not only the decorative patterns but also the innovation and development of the manufacturing process, witnessed the brilliant history of ethnic characteristic lacquer wares. With the development of modern technology and the improvement of living standards, the demand for the lacquerware crafts tends to be weak, therefore it's failed to realize the heritage and development, innovation integrated into inheritance. Considering the complexity and time-consuming of the manufacturing process of ethnic lacquerware crafts, this paper aims for the greater development of the ethnic characteristic lacquer wares through the development of technology, the innovation of materials, the richness of patterns, and the practicability of the expansion, to make an important step on the international achievement.
\end{abstract}

\section{Keywords}

Ethnic Characteristics, Lacquerware Crafts, Lacquerware Development, Lacquerware Creation

\section{Origin and Development of Lacquerware Crafts}

The natural liquid of varnish tree is the main source of raw lacquer. Lacquerware, as the name suggests, is composed of the combination of lacquer and wares. The crafts and the decoration articles with the surface decorated with lacquer, are so-called "lacquerware". Huang Cheng, a prominent folk decoration artist in the Ming Dynasty, commented on lacquerware in his monograph Xiushilu (Records of Lacquering), "Covered with lacquer is for the strong quality 
and the brilliant surface of the wares". Lacquerware belonged to an important invention in chemical technology and arts and crafts in ancient China.

The origin of lacquerware can be traced back to the Neolithic Age. Through the Shang and Zhou Dynasties to the Ming and Qing Dynasties, the Chinese lacquer wares have been developing continuously. The prehistoric lacquer wares have shown its prospects. Chinese lacquerware culture was rooted in the Neolithic Age, basing on the human material civilization and cultural development. As early as 6000 years ago, in the Hemudu Culture Age, the lacquerware made at 7000 years ago has been found. A red lacquered wood bowl opened the door of Chinese lacquerware and made China become the first country of integrating lacquer on wares for decoration.

The lacquerware of Shang and the Western Zhou Dynasties came into mature gradually. The Chinese lacquer wares in Xia, Shang and the Western Zhou Dynasties were still in their embryonic periods in the history of lacquer development. The bronze smelting and casting technology, the utilization of bronze ware, and the planting and raising of man-made lacquer forest provide solid material foundation and technical conditions for the development of lacquerware handicraft industry. The colored drawing, gold and silver decals, inlaid jades and other lacquer wares emerged in early time show that the cultural and artistic language system of raw lacquer has shown its initial form. With the increased styles and patterns of lacquerware, the splendid lacquerware was converted into a symbol of luxury eventually.

The luxurious and gorgeous lacquerware emerged in the Spring Autumn and Warring States Periods. The Spring Autumn and Warring States Periods were defined as the growing periods of the Chinese ethnic lacquerware in its development history, and with the wooden-cored lacquer wares became into the mainstream, the large-scale appliances and musical instruments were also upgraded in color, pattern and decoration technology. With the master of more methods to make lacquer embryos, together with the excellent technology, people can produce many new styles of lacquer embryos, which marks that the Chinese embryos making technology of lacquerware reached a new level.

Compared with the Spring Autumn and Warring States Periods, the lacquerware of Qin Dynasty was more standard and exquisite, with its rich variety and increased output, which was widely used in all aspects of social production. The Qin Dynasty was a transitional period during the development of lacquerware in China. Because of its short history, the number of lacquerware inherited from this period was much less. While compared with the Warring States period, the lacquerware in the Qin and Han Dynasties was utilized more universally with the form of furniture more often, shown with certain epochal characteristics in the manufacturing process, decorative patterns and color decoration. The rich themes of lacquer drawing leaded to the research and production of large-scale lacquerware. On the one hand, the themes about wise men and sages were integrated into social life. On the other hand, taking the real life as the theme, people innovated the decorative patterns, such as cloud pattern, mountain pattern and 
some other decorative patterns. Double sides colored screens belonged to one kind of large lacquerwares in Han Dynasty. The innovation of the decoration crafts on "gold and silver colored drawing" and "inlaid gold" made the lacquerware technology more and more used in the creation of large murals (Deng, 2011). The innovation of manufacturing process, the rich types of lacquerware and the clear division of production made the lacquerware crafts reach the summit.

The prosperous lacquerware period occurred in Han Dynasty. With the crafts inheritance from the Warring States Period and Qin Dynasties, the Han lacquerwares took a new step forward. The lacquerware style in this period tended to be analogous, and the number of lacquerware increased. With the producing areas of lacquerware increased dramatically, the lacquer crafts have developed to be the most prosperous unprecedentedly. In the Western Han Dynasty, the government-run lacquerware industry set up a systematic and rigorous production organization to ensure the development of lacquer technology in depth.

The lacquerware was increasingly sophisticated in Wei and Jin Dynasties, and during the period of the Three Kingdoms, the Two Jins, the Northern and Southern Dynasties, it developed more diversified, with various decorative patterns including all kinds of birds, animals and insects, dancing, music, hunting and character narration etc. During this period, many achievements have been made, such as the application of lithargite and the invention of green varnish.

It showed the sumptuously decorated lacquerware in the Sui and Tang Dynasties. The decoration of lacquerware in Tang Dynasty was magnificent and sumptuous, with various new process and techniques come out one after another. The expensive lacquerware with inlaid gold and silver decoration appeared, which made the lacquerware of this period gorgeous and exquisite with distinguished characteristics of times.

The lacquerware made in Song and Yuan dynasties was excellent. In the Song Dynasty, the polished lacquer refinement technology and the lacquered polishing workmanship on wares were invented, on the basis of raw lacquer made by lithargite in Wei Jin Southern and Northern Dynasties, which pushed the decorative function of lacquer to the acme. In the Song Dynasty, the most characteristic workmanship for the lacquer wares was embodied on their lacquerware embryo made of circles, with long durability.

The Ming and Qing lacquerware was with thousands of patterns and distinguished decorations. The varieties of lacquerware in Ming Dynasty developed rapidly, on the basis of the lacquerware development in Song and Yuan Dynasties, in which the most developed and the largest number of lacquer wares in this period was the carved lacquerware. Qing Dynasty was considered to be the heyday of lacquerware development, with the distinguished characteristics compared with the Qin and Han lacquerware. During this period, private lacquer factories sprang up all over the country with many kinds of lacquerware crafts produced, which helped to form the characteristic lacquerware of Qing Dynasty. For example, Fuzhou bodiless lacquerware and Yangzhou inlaid lacquerware etc. 
The Chinese lacquerware culture has a long history, with profound cultural basis and historical background, including the inlaid and gilded of Gold on handicrafts, which had a far-reaching impact on Japan and other places. Some scholars studied the loss of ancient lacquerware culture and indicated that lacquerware culture originated in China but prevailed in Japan, in which the main reasons are summarized as follows: 1) The decorative functionality and patterns are too traditional. For example, the repeated occurrence of dragon and phoenix in patterns did not match the modern aesthetics and decorative effects of modern living rooms any more (Deng, 2011). The practicability of lacquerware crafts came to be weakened, accompanying with the inferior application of crafts, decorative ornaments and wares than Japan. Suppose we could better presentation them in the eyes of the public, which will help win a great market, so as to achieve the more excellent development. 2) The small market share, uncertain development prospects and the shortage of professionals, coupled with the transformation and promotion of people's ideas in the new era, which make the development of traditional Chinese lacquerware was at a standstill, even lagged behind Japan. Nowadays, the development of traditional lacquerware in technology, innovative materials, rich and decorative patterns, practicability, and the enlarged markets and other aspects make Chinese traditional lacquerware took the new lead step by step. After a long period of practice, people have accumulated richer experience in the selection and production of lacquerware embryo, the allocation and practicability of paints, and the drawing and combination of lacquer decoration etc. They have developed lacquerware crafts into a special workmanship and promoted them into a high level, forming a unique lacquer crafts with Chinese characteristics. The invention of Chinese lacquerware is taken as one of the great contributions to the world made by the Chinese people.

\section{Study on Large Lacquerware Crafts with Ethnic Characteristics}

\subsection{The Development Tendency of Large Lacquerware Crafts with Ethnic Characteristics}

Professor Qiao Shiguang ever mentioned in Lacquer Art, "The lacquer art includes three parts of lacquerware, stereo painting and lacquer painting" (Zheng, 2018), and the three parts are more focused on their aesthetics rather than its practicality in the process of development and creation. Meanwhile, the development prospects of lacquerware crafts seemed not optimistic for the over complicated production process, the excessively single decoration, and the insufficient talented personnel.

The lacquerware, such as the red lacquered bowl, used in early China seems practical to some extent, which was widely used in various aspects of social life because of its low cost and simple production process. However, with the availability of porcelain, there was significant reduction in the practicability of lacquerware, and more of them were used as private and collected objects for 
princes and nobles (Zheng, 2018). Due to the impact of historical wars, it's the lack of consideration on the status of lacquerware, which led to the stagnation of the development of lacquerware crafts. On the contrary, lacquerware is widely used in Japan, the country across the sea from China, ranging from wares to large lacquerware screens, tables and chairs, with their strong practicability. In the midst of the development tendency, China listed Fuzhou bodiless lacquerware, Chengdu lacquerware, Yangzhou inlaid lacquerware and Pingyao polished lacquerware in the list of intangible cultural heritage, to forge them into the pillar industries in the local area and bring the considerable economic benefits. After the New China was founded, the political, economic and cultural get more and more attention, while the art was still at a stagnant stage to a certain extent. The loss of national culture has had a negative impact on the development of large-scale lacquerware with national characteristics. So in the future, for the lacquerware craft market, it should take national characteristics as the fundamental key and highlight the essence of traditional Chinese culture.

\subsection{Research Direction of Large Lacquerware Crafts with Ethnic Characteristics}

As the first country that discovered and processed lacquer, China has a unique research on lacquer handicrafts. Compared the Chinese lacquerware with the traditional Meticulous paintings, we will find some similarities from the two different workmanship ways, that is, whether lacquerware or lacquer painting, their creation style is unique, emphasizing the drawing of auspicious patterns, retaining the traditional painting workmanship in the manufacture process to some extent, stressing "bright, polished, smooth", emphasizing the "lacquer soul" of lacquerware. For example, the Fuzhou bodiless lacquerware, seems to have a place in both modern aesthetics and modern market, by its advantages of convenience, delicate and beauty.

As the wisdom crystallization of industry and art, the lacquerware crafts not only pursue the beauty of form, but also pay attention to the beauty of their internal artistic conception. The Chinese ethnic culture is extensive and profound with a long history, and the contemporary research and creation of Chinese lacquerware crafts were paid attention to the aesthetic unity of forms and artistic conception. The modern lacquerware crafts cater to the social market demand, with the innovation and development implemented from two aspects of decoration and practicability, with their decorative patterns embodying the aesthetic harmony and unity of forms and artistic conception. With the exchange and collision of traditional culture, traditional lacquer art and modern lacquer art, a number of new large lacquerware emerged, which was different from the forms of ancient screens and murals. For example, the lacquered screens and murals in the Beijing Great Hall of the People, Story by the Minjiang River, Mountain Scenery, Baodao Butterfly Emotions and a series of other works are both innovative achievements produced under the concept of inheriting lacquerware with national characteristics. The modern lacquerware should achieve the artistic 
conception of "painting in poetry, poetry in painting" as other arts do (Ge, 2009). The unique artistic effect and charm of the lacquerware itself, as well as its properties of anti-corrosion and anti-acid \& alkali, should be highly harmonious with modern indoor furnishings. In the production of lacquerware, with the interesting and novel combination of such artistic forms like "Chinese paintings", "engravings" and "oil painting", which would greatly expand the expressiveness and appeal of lacquerware crafts, enrich the characteristics of lacquerware itself and enhance its artistic value.

\subsection{The Innovation of Large Lacquerware Crafts with Ethnic Characteristics}

Until now China has been the earliest country to use lacquer for color matching, painting, combination with wares for decoration. China's lacquerware production has a long history and has made brilliant artistic achievements. With the development of modern science and technology and the improvement of people's living standards, the lacquerware has gradually faded from the circle of daily life. How to universalize the lacquerware with strong Chinese ethnic cultural characteristics and how to inherit and develop the essence of lacquerware will be taken as an important theme of contemporary research.

Innovation does not mean the abandon of tradition, but aims for the combination of traditional crafts with modern technology, incorporated with the innovative thoughts and creative materials, so as to create a number of lacquerware crafts with ethnic characteristics with modern aesthetics: 1) combining traditional handicraft with modern development ideas, merging the development of handicraft market with tourism industry, so as to integrate traditional lacquerware crafts into modern life in a large degree; 2) combining traditional arts with modern arts, which coordinates with each other and collides with a unique spark; 3) combining traditional craftsmanship with contemporary crafts, to realize the inheritance of the spirit of traditional craftsmen; 4) considering their unique and complex manufacturing process, and their rich decorative patterns and colors, on this basis, the lacquerware crafts with ethnic characteristics should be combined with modern technology and are simplified constantly on the production process, enriched decorative patterns and innovative matching color, so that the ethnic lacquerware crafts will be popular among the general public of all ages, be developed further and better in display, decoration and art.

\section{The Creation of Large Lacquerware Crafts with Ethnic Characteristics}

\subsection{Manufacturing Process of Large Lacquerware Crafts with Ethnic Characteristics}

Lacquerware is the main lacquer art form of expression, followed by lacquer painting and carved lacquerware. The earliest lacquerware was mainly applied to daily life, and in modern society the collection function comes to be the major 
trends. Undergoing several generations of changes and a series of reform and development, the lacquerware shows the different characteristics on the technology and techniques in different periods, sometimes with the regional difference.

The raw materials of lacquerware came from the sap extracted from the trees in nature, which is called raw lacquer, or known as Chinese lacquer, with the role of decoration and protection for utensils, it has the characteristics of moisture resistance, high temperature resistance, corrosion resistance and so on. Utilizing the different visual effects achieved in decoration, the materials will be decorative, flexible, creative, compatible and malleable. The manufacture process of the natural raw lacquer includes lacquer selection, filtration, air-curing, sun-curing and fine filtration. In the Xia and Shang Dynasty, there emerged a new technology of embossed lacquer, which is to stack three-dimensional patterns by making sticky paint accumulated repeatedly. At the same time, it combined many kinds of inlaid materials, such as mother-of-pearl, gold and silver in innovation, and shaped beautiful patterns after design (Zheng, 2001). By the Song and Yuan Dynasties, the gorgeous decoration of lacquerware was faded, mainly specializing in the elegance and simplicity of carving lacquer, to show the craftsmen's love for the crafts from the notches. The lacquer arts in Ming and Qing dynasties were kept developed in a state of progress continuously, by consistent absorbing of the characteristics of various nationalities and excellent foreign culture, which added the new features for the development of lacquerware. With the lacquer technology is widely used in furnishings, the demand has been changed. In the process of exploration, more and more decorative techniques came into being, such as the mottled patterns and the inlaid patterns etc.

\subsection{Composition Approaches of Large Lacquerware Crafts with Ethnic Characteristics}

Faced with the new era and new ideas, the traditional crafts have also been changed. The workmanship and the techniques should be formulated with new ideas, with the rejection of meaning devoid and the equipment of a new implication. With the rich forms, the lacquer is inclusive for any kind of material no matter for the lacquer painting or the lacquer ware, it will realize the ever-changing decorative effects.

With the rapid development of economy, in order to avoid the eyes kept from recognizing for an instant, we should understand as soon as possible that we should not only pay attention to the proficiency of decoration and workmanship during the production of lacquerware, but also endow the lacquerware with internal ideas in the process of creation, and give it an understanding of life, people and all creations in different periods, rather than simply showing skills and crafts (Zhang, 2015). The minority nationality has their own traditional culture and spiritual pursuit as well as aesthetic characteristics, so that the extending of the excellent traditional culture should be the key point, with the integrating of spiritual pursuit and other characteristics into lacquerware and the endowment of life into each lacquerware, which will be the long-term strategy: 
1) The combination of lacquerware and modern scientific and technological means. Ancient traditional lacquerware production asked for the strict environmental conditions, especially for the temperature, which will take a long time to finish. At present, with the merging of modern scientific and technological means, the indoor temperature can be adjusted automatically, which make the work efficiency improved greatly. 2) Innovation is an indispensable means at present. Although the application of the science and technology shortens the production cycle of lacquerware, the charm of traditional hand crafted lacquerware cannot be replaced, including the replacement of its own style and characteristics, and it is difficult to erase the good implication from the persons. Therefore, we should innovate, understand and explore the ideological and aesthetic characteristics embodied by people in this era, integrate them into production, and endow them a new life under the condition that the traditional characteristics of lacquerware are well kept.

We should take the initiative to understand the traditional crafts, consistently attempt and explore new materials and crafts in the creation, and then try to apply the new materials and crafts into the creation. Create new patterns with new aesthetics and ideas, and gradually change the original realistic style to the abstract, making the content simple but without the deficiency of details and implications. The manuscript part ahead of lacquerware production can be completed by modern scientific and technological means to speed up the efficiency, by which more time will be saved and spent on the verification of the feasibility, till the standard will be achieved for painting and drawing (Liu, 2014). The impression of Yin and Yang concave and convex will be formed by the embossed lacquer on the designed traces, ground to smooth after one more layer coating paint, furthermore came to the process of 10 times grinding after sealing paint. This process may be unpredictable, and we should regard it as the source of inspiration.

\section{Conclusion}

In view of its ways of development and creation, this paper probes into the origin and historical evolution of lacquerware, explores the trend and research direction of lacquerware crafts in its development process, summarizes the essence of art, traditional skills and craftsman spirit that should be paid attention to in modern development and innovation, and puts forward the creative approaches with traditional cultural value and inheritance significance.

\section{Acknowledgements}

This project is funded by the graduate innovative scientific research project (grant number: CX2019SZ07) of southwest university for nationalities and this project is funded by the undergraduate innovation and entrepreneurship training program of southwest university for nationalities (project number: X201810656008). 


\section{References}

Deng, L. L. (2011). Thoughts on Current Status and Development Countermeasure of Yangzhou Lacquerware. Fine Arts Journal, No. 8, 74-76.

Ge, H. (2009). Inheritance and Development of Modern Lacquer Art v.s. Traditional Lacquer Art. Soochow: Soochow University.

Liu, F. L. (2014). The Combination of Lacquer Art and Minority Art. Art Science and Technology, 27, 93-94 + 99 .

Zhang, M. L. (2015). Trial Discuss On the Inheritance and Development of Modern Lacquer Art to Traditional Lacquer Art. Art Science and Technology, 28, 188.

Zheng, X. (2001). Brief Analysis on the Inheritance, Development and Innovation of Lacquer Painting. Nanfang Wenwu, No. 2, 122-124.

Zheng, Y. T. (2018). Research on the Development of Modern Lacquer Art. Art and Literature for the Public, No. 3, 134. 